\title{
MODIFICATIONS PHYSICO-CHIMIQUES DES CONSTITUANTS DU LAIT AU COURS DE LA CONCENTRATION EN PRÉSENCE DE SIROP DE SUCRE
}

\author{
par \\ Michel POLONOVSKI
}

La grande diffusion du lait concentré sucré dans l'alimentation actuelle des nourrissons avait déjà permis, par de simples constatations statistiques, d'affirmer que le lait condensé provoque moins de diarrhées cholériformes que le lait ordinaire et nombre de puériculteurs le considèrent même comme supérieur au meilleur lait de vache frais. D'une étude suivie et détaillée que nous avons publiée avee P. Gelté l'an dernier (1) nous concluions qu'il existe réellement entre le lait condensé sucré et le lait de vache sucré au même taux une différence notable de digestibilité. En effet :

$1^{\circ}$ Les nourrissons ne peuvent supporter, additionnée au lait frais, une dose de sucre égale à celle qu'il digère sous forme de lait concentré sucré, sans présenter des phénomènes d'intolérance (vomissements, diarrhée).

$2^{\circ}$ Dès la fin du troisième mois et surtout après le sixième, l'augmentation de poids des enfants devient nettement plus importante lorsqu'ils sont nourris au lait condensé sucré (2).

Ces résultats diététiques demandaient une explication rationnelle : quelle modification heureuse la concentration du lait, en présence de sirop de sucre, pouvait-elle imprimer aux constituants de cet aliment primordial pour influer ainsi sur sa digestibilité ?

Nous avons essayé de préciser la cause de ces variations et à cet effet nous avons étudié comparativement la tension superficielle, le pouvoir-tampon, la viscosité et enfin la floculabilité des différents laits. Il nous a semblé intéressant de suivre un même lait au cours de la concentration, afin de pouvoir observer l'influence de cette opération. Aussi avons-nous effectué les déterminations sur la série des laits suivants : lait frais, lait pasteurisé, lait pasteurisé + sucre, lait en fin d'admission dans les appareils de concentration dans le vide, lait en cours de condensation, lait en fin de condensation (2).

\section{A. Essais préliminaires.}

$1^{0}$ Conditions à réaliser pour que les résultats soient comparables entre eux.

(1) De l'emploi comparé du lait concentré sucré et du lait de vache frais dans l'alimentation du nourrisson. Echo médical du Nord (3), t. V, 1936, p. 805-819.

(2) Les essais ont été faits, comme d'ailleurs tout le travail expérimental que nous publions aujourd'hui, avee le lait concentré sucré que la Société Nestlé a eu l'obligeance de mettre gracieusement à notre disposition. 
La première condition pour obtenir des résultats comparables est de ramener les composants des différents laits à une concentration voisine de celle qu'ils ont dans le lait ordinaire, exception faite pour le saccharose. Nous avons forcément dû choisir comme lait de base un de ceux que l'addition de sucre avait déjà dilué ; nous avons donné la préférence au lait pasteurisé sucré, la pasteurisation ne modifiant pas sensiblement la concentration. D'autre part, le lait frais et le lait pasteurisé simple ont également dû être étudiés après addition d'une quantité de sucre égale à celle qui est utilisée dans la fabrication du lait concentré.

Enfin, les mesures ont été pratiquées dès l'arrivée des échantillons au laboratoire, l'ouverture des boîtes précédant immédiatement la dilution et la mise en route des expériences.

\section{$2^{\circ}$ Détermination des taux de concentration.}

Nous avons pris comme référence les éléments dont les variations sont uniquement imputables à la concentration et qui ne peuvent pas subir de modifications sous les influences extérieures. L'azote total et les matières réductrices totales ont retenu notre attention. Nous avons utilisé pour le premier la méthode de Kjeldahl, pour les secondes la méthode d'oxydation sulfochromique (indice chromique. de Polonovski).

TABLEAU I.

\begin{tabular}{ccc}
$\begin{array}{c}\text { N total } \\
\text { en grammes } \\
\text { par litre }\end{array}$ & $\begin{array}{c}\text { Indice } \\
\text { ehromique } \\
\text { total }\end{array}$ & $\begin{array}{c}\text { Concentration } \\
\text { par rapport } \\
\text { au témoin } 3, \\
\text { calculé d'après } \\
\text { le taux N }\end{array}$ \\
4,48 & 67 & 1,23 \\
4,48 & 73 & 1,23 \\
3,64 & 251 & 1 \\
\hline 12,46 & 852 & 3,4 \\
12,74 & 900 & 3,5 \\
17,01 & 1.000 & 4,6
\end{tabular}

1. Lait frais . . . . . . . . .

2. Lait pasteurisé ..........

3. Lait pasteurisé + sucre ...

4. Lait fin d'admission .......

5. Lait en cours de condensation $\ldots \ldots \ldots \ldots \ldots \ldots$

6. Lait en fin de condensation

Le tableau nous montre que la pasteurisation simple ne modifie pas sensiblement la concentration, puisque la teneur en azote est identique dans les laits frais et pasteurisé. Le lait pasteurisé sucré est au contraire plus dilué, ce qui s'explique par l'addition de saccharose sous forme de sirop, et ce qui justifie notre choix de ce lait comme étalon de concentration. D'autre part, les résultats donnés par la mesure de l'indice chromique total concordent assez bien avec les dosages d'azote, tout en restant dans l'ensemble légèrement inférieurs. La pasteurisation n'influe pas non plus d'une façon notable sur le taux des matières réductrices. L'oxydation 
intégrale du saccharose explique la brusque augmentation de l'indice à partir du lait $n^{\circ} 3$.

\section{$3^{\circ}$ Quantité de saccharose à ajouter au lait frais.}

Il était possible de la déterminer en dosant dans le lait pasteurisé sucré les sucres réducteurs avant et après hydrolyse, par la méthode de Bertrand ou celle de Baudouin. Nous avons écarté ce procédé, car l'ébullition, au cours du premier dosage, peut, dans une certaine mesure, hydrolyser le saccharose, ce qui donne une erreur par défaut dans le résultat final.

Aussi avons-nous préféré la méthode suivante : on mesure la déviation polarimétrique A donnée par le lait pasteurisé simple, puis celle du lait pasteurisé sucré ; on corrige ensuite celle-ci en la multipliant par l'inverse du rapport des concentrations en azote : soit B la valeur de la seconde déviation ainsi obtenue. La différence B-A indique la déviation due au saccharose, rapportée au lait pasteurisé, et par suite la quantité de sucre ajoutée à ce lait.

Le résultat de ce calcul pour notre lait fut le suivant :
$\mathrm{A}=23^{\circ} 27$
$\mathrm{B}=5^{\circ} 50$.

$\mathrm{A}-\mathrm{B}=17^{\circ} 37$, ce qui correspond à $132 \mathrm{gr}, 4$ de saccharose par litre.

\section{B. Viscosités.}

Les mesures ont été faites au viscosimètre Beaumé ; nous avons opéré à la température ordinaire pour éviter l'influence possible de la chaleur sur les composants du lait. Dans tous les cas nous avons vérifié les résultats en déterminant la vitesse d'écoulement au moyen d'un stalagmomètre.

TABLEAU II.

$\begin{array}{ccc}\text { Température 17.18 } & \text { Vidité } & \text { Vitesse } \\ \text { Barbey } & \text { Englé } & \begin{array}{c}\text { d'écoulement } \\ \text { (nombredegouttes } \\ \text { par minute) }\end{array}\end{array}$

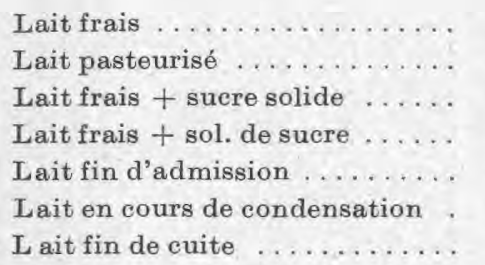

2.677

2.417

1,061

90

1. 644

1,076

2. 018

1,133

86

2. 055

1,147

1.500

1,107

1. 550

1,105

1,168

78

84

-

72

73

\section{Tension superficielle.}

Nous avons utilisé la méthode suivante : Quand dans un tube étroit d'ouverture donnée (3 à $4 \mathrm{~mm}$.), les gouttes qui se forment à l'extrémité sont soumises à une pression constante, la tension superficielle appliquée à la goutte qui se détache, tension qui 
contrebalance le poids de la goutte, est liée à ce dernier par la relation

$$
\begin{aligned}
& \mathrm{P}=\mathrm{K} \cdot 2 \pi \cdot \mathrm{Ar} \\
& \text { ou, en posant K. } 2 \pi . \mathrm{r}=\mathrm{K}^{\prime} \\
& \mathrm{P}=\mathrm{K}^{\prime} \mathrm{T}
\end{aligned}
$$

Connaissant $T$, tension superficielle de l'eau à $20^{\circ}(72,8)$, on étalonne l'appareil avec l'eau, et on connaît ainsi la constante $\mathrm{K}$. Voici les résultats de nos mesures:

Température $18^{\circ}$

Premier essai

Lait frais

Lait pasteurisé

51,33

53,15

56,12

55,47

55,35

56,36

56,33

Lait fin de condensation ............
Deuxième essai (sur un autre lot de boîtes )

51,62

53,72

55,98

55,66

56,46

56,40

\section{Pouvoir-tampon.}

Nous avons exprimé le pouvoir-tampon par la quantité d'acide normal qu'il faut ajouter à un litre de lait pour passer du virage de la phénolphtaléine $(p \mathrm{H} 8,5)$ au virage de l'hélianthine $(p \mathrm{H} 4,5)$.

Quantité de
$\mathrm{NaOH} \mathrm{N} / 50$
nécessaire
pour atteindre
le virage de la
phénolphtaléine

(Sur $2 \mathrm{~cm}^{3}$ de lait)

\section{Quantité de $\mathrm{SO}^{4} \mathrm{H}^{2} \mathrm{~N} / 50$ nécessaire} pour revenir au virage de l'hélianthine

Lait frais + sol. de sucre ...

Lait pasteurisé + sol. de sucre $\ldots \ldots \ldots \ldots \ldots \ldots$.

Lait fin d'admission .......

Lait en cours de condensation $\ldots \ldots \ldots \ldots \ldots$.

Lait fin de cuite ........ 1,50
4,85

1,25

1,40
$10 \mathrm{~cm}^{3} 40$

104

107

82,5

100

101,5

Pouvoirtampon

Deuxième lait :

$$
(\mathrm{NaOH} \mathrm{N1} / 0) \quad(\mathrm{HCl} \mathrm{N} / 10)
$$

(Pour $5 \mathrm{~cm}^{3}$ )

\section{Lait frais}

$$
0,65
$$

Lait pasteurisé + sol. de sucre.

0,65

5,90

Lait pasteurisé + sucre solide . .

Lait fin d'admission ......... 0,50

Lait en cours de condensation .. 


\section{E. Floculabilité.}

$1^{\circ} \mathrm{pH}$ critiques, zones de floculation.

Nous avons mesuré le $p \mathbf{H}$ colorimétriquement dans le lactosérum, après filtration de la caséine. Nous n'avons retenu dans les séries de tubes que ceux dans lesquels la coagulation était totale de façon à éviter l'erreur due à l'adsorption du colorant par la caséine restante. Il en résulte que les zones de floculation ainsi déterminées paraissent un peu plus étroites qu'elles ne sont en réalité ; mais ce qui importe, c'est que l'allure générale se conserve et que les résultats restent comparables entre eux.

Les laits ont été préalablement additionnés d'eau distillée neutre, de façon à rendre la floculation plus visible. L'acide utilisé a été l'acide chlorhydrique $\mathbf{N} / \mathbf{1 0}$.

Nous devons d'abord rappeler une série de remarques qui nous paraissent indispensables à la compréhension et à l'interprétation des résultats précédents et de ceux qui suivront.

a) Si on ajoute au lait une faible quantité d'acide de telle sorte que son $p \mathbf{H}$ diminue, mais reste toutefois plus grand que 5,3, il n'y a pas de floculation immédiate.

b) Si on ajoute en une seule fois, brusquement, une quantité d'acide telle que le $p \mathrm{H}$ tombe au-dessous de 4 : la floculation ne se produit pas non plus.

c) Si on ajoute l'acide goutte à goutte, on obtient, en dessous de $p H 5,3$, une floculation instantanée, très nette, le précipité se rassemblant rapidement au fond du tube. Cette précipitation immédiate se produit dans une zone de $p H$ limitée par deux $p H$ critiques, indiqués pour les différents laits dans le tableau suivant. Entre les deux $p H$ critiques se trouve un $p H$ optimum, pour lequel la vitesse de sédimentation de la caséine est la plus grande, c'est-à-dire où la floculation est la meilleure.

Limite inférieure Limite supérieure de la zone de floculation

Lait frais $\ldots \ldots \ldots \ldots \ldots \ldots$

Lait + sirop de sucre ..........

Lait + sucre solide ..........

Lait pasteurisé .............

Lait pasteurisé + sirop de sucre ...

Lait fin d'admission . . . . . . . . . .

Lait en cours de condensation ....

Lait fin de cuite .............

$\begin{array}{ll}4,2 & 5,3 \\ 4,1 & 5,0 \\ 4,4 & 5,25 \\ 3,95 & 5,0 \\ 4,0 & 4,8 \\ 4,25 & 5,0 \\ 4,0 & 4,7 \\ 3,95 & 4,65\end{array}$

d) Si l'on augmente la quantité d'acide, on remarque qu'endessous du $p H$ critique inférieur, les grains de caséine deviennent de plus en plus fins, et que, finalement, la caséine se met en suspen- 
sion. Cette suspension n'est d'ailleurs pas stable; si le tube reste abandonné à lui-même, la caséine se dépose de nouveau, le liquide surnageant restant trouble. Si le contact de la caséine et de l'acide dans la zone de floculation a été prolongé, la vitesse de sédimentation est très augmentée $\left(30^{\prime}\right.$ au lieu de $\left.65^{\prime}\right)$, mais la vitesse reste toujours très inférieure à celle que l'on observe dans la zone de floculation.

$2 \circ$ Floculation en présence de lab-ferment.

La floculation sous l'influence de la présure a tout d'abord été étudiée à la température de $37^{\circ}$, à $p H$ voisin de 3,0 . L'acide chlorhydrique nécessaire pour obtenir ce $p H$ était naturellement ajouté en une seule fois pour éviter la coagulation immédiate, ainsi que nous l'avons signalé dans le paragraphe précédent.

Temps nécessaire à la floculation complète en minutes

Lait frais . . . . . . . . . . . . . 10

Lait frais + sucre solide ........

Lait frais + sirop de sucre $\ldots \ldots \ldots$. $\quad 20$

Lait pasteurisé ............ $\quad 30$

Lait pasteurisé + sucre solide .... $\quad 35$

Lait pasteurisé + sirop de sucre ... $\quad 45$

Lait concentré sucré ........... 45

Nous avons ensuite fait agir le lab-ferment dans des milieux d'acidité différente, le $p \mathrm{H}$ de 3,0 qui était celui des déterminations précédentes, n'étant réalisé physiologiquement que par le mélange d'un faible volume de lait et par une grosse quantité de liquide gastrique.

Nous avons fait une série de mesures sans addition d'acide ( $p$ H voisin de 7 ), puis en présence de $0 \mathrm{~cm}^{3} 25$ et de $0 \mathrm{~cm}^{3} 15$ de $\mathrm{HCl} \mathrm{N/10.}$

Temps nécessaire à la floculation

(en minutes)

Lait frais . . . . . . . . . . . .

Lait pasteurisé ..............

Lait pasteurisé + solution de sucre

Lait pasteurisé + sucre solide ....

Lait concentré

$3^{\circ}$ Relation entre la floculabilité, la viscosité et la tension superficielle.

Il nous a semblé intéressant de vérifier si les différences observées entre les floculabilités des laits n'étaient pas en rapport avec les variations de la viscosité et de la tension superficielle.

Nous avons tout d'abord modifié la viscosité des laits par l'addi- 
tion de sirop de gomme du codex : gomme blanche, 100 gr. ; sucre blanc, 560 gr. ; eau distillée, 340 grammes.

Le tableau suivant donne les résultats obtenus par ce procédé.

Viscosité à $18^{\circ}$ Variation en $\%$

Lait pur . . . . . . . . . . .

$\begin{array}{ll}1,083 & \\ 1,140 & +5,2 \\ 1,201 & +11 \\ 1,190 & +10\end{array}$

Pour abaisser la tension superficielle, nous avons utilisé une solution saturée à $18^{\circ}$ de sels biliaires.

Tension superficielle

à $20^{\circ}$

Lait pur ..................

Lait pur $+2 \%$ sol. sels biliaires ...

Lait pur $+4 \%$ sol. sels biliaires ...
44,32

43,74

38,65
Variation en $\%$

$-1,3$

$-12,77$

Nous avons étudié comparativement la floculabilité des laits frais et pasteurisé sous l'influence de la présure en milieu acide $(p \mathrm{H}$ voisin de 3,0$)$ et à $37^{\circ}$, avant et après addition d'une part de sirop de gomme et d'autre part de sels biliaires.

\begin{tabular}{|c|c|c|}
\hline Lait frais & $\begin{array}{l}\text { Temps en minutes } \\
\text { sol. }+4 \% \text { de sol. } \\
\text { de sels biliaires }\end{array}$ & $\begin{array}{l}\text { Sol. + } 25 \% \text { de } \\
\text { sirop de gomme. }\end{array}$ \\
\hline 10 & 5 & 15 \\
\hline 10 & 5 & 15 \\
\hline 10 & 5 & 15 \\
\hline 10 & 5 & 15 \\
\hline
\end{tabular}

$1^{\text {er essai }} \ldots \ldots \ldots \ldots \ldots \ldots$
$2^{\text {e essai } \ldots \ldots \ldots \ldots \ldots \ldots}$
$3^{\text {e }}$ essai $\ldots \ldots \ldots \ldots \ldots \ldots$
$4^{\text {e }}$ essai $\ldots \ldots \ldots \ldots \ldots \ldots$

Lait frais

Lait pasteurisé

30

30

30

$3^{\text {e essai } \ldots \ldots \ldots \ldots \ldots}$

Lait frais

10 sol. $+25 \%$
sirop de gomme

15

$$
\begin{gathered}
\text { Sol. + sirop } \\
\text { de sucre }
\end{gathered}
$$

20

\section{Lait pasteurisé}

30

Lait pasteurisé

30
Lait pasteurisé $+25 \%$ sp de gomme

60

60

65

Sol. $+25 \%$

sirop gomme

60

Sol. + sirop

sucre

45

La floculabilité varie donc en sens inverse à la fois de la tension superficielle et de la viscosité. Ainsi qu'il ressort des deux dernières séries de chiffres, la diminution de la floculabilité est du même ordre 
lorsqu'on remplace le sirop de gomme par du sirop simple; cela s'explique aisément par les variations presque identiques de la viseosité observée dans les deux cas.

\section{F. Interprétation des résultats.}

Nous pouvons résumer de la façon suivante l'ensemble des résultats que nous avons obtenus. Par rapport au lait pasteurisé additionné de sirop de sucre, - lait dont il est le plus voisin par la. composition et les opérations subies -, le lait concentré sucré présente une viscosité et une tension superficielle augmentées, et une floculabilité diminuée, le pouvoir-tampon ne subissant qu'une variation faible, d'ailleurs inconstante. Ces différences s'accentuent naturellement quand on compare le lait concentré au lait frais dont il dérive.

Il est difficile d'établir un lien direct entre toutes les modifications observées et la meilleure utilisation du lait chez le nourrisson. Il est cependant intéressant de considérer plus particulièrement la floculabilité par la présure en milieu acide. L'influence de la coagulation du lait sur sa digestion est un fait bien connu; un lait qui se coagule rapidement en formant dans l'estomac un caillot massif, comme le lait de vache pris en grande quantité, est beaucoup moins toléré qu'un lait dont la coagulation se ferait plus lentement et aboutirait à la formation d'un grand nombre de petits caillots. L'action du suc gastrique est naturellement très facilitée dans ce dernier cas, et l'évacuation gastrique elle-même doit se faire différemment. On pourra nous objecter que la floculabilité du lait concentré n'est que peu retardée par rapport à celle du lait pasteurisé sucré, qu'il ne s'agit que de quelques minutes. A cela nous répondrons que, quoique nos mesures aient été faites dans des conditions qui s'éloignent plus ou moins des conditions physiologiques, nous avons cependant, dans tous les cas, observé une différence dans le même sens, et que l'écart a été d'autant plus grand que nous nous rapprochions davantage de ce qui se trouve réalisé dans le milieu stomacal.

L'influence de la viscosité et de la tension superficielle nous paraît être plutôt indirecte; ces constantes physiques n'interviennent très probablement que dans la mesure où elle modifie la floculabilité. Les chiffres montrent d'ailleurs que les variations de celle-ci ne peuvent s'expliquer uniquement par celles des deux premières.

Il nous semble en définitive plus sage de considérer les modifications moins en elles-mêmes qu'en ce qu'elles constituent des manifestations de changements plus profonds que ceux que nos premières investigations pouvaient déceler. Il est certain que les opérations 
de concentration du lait en présence d'une grosse quantité de sucre ne laissent pas intacts ses constituants, et plus spécialement les substances protidiques. Nos résultats n'ont aucunè prétention à avoir déjà résolu ce problème, trop heureux si nous avons, en soulevant un coin du voile, jeté sur lui quelque clarté.

\section{ACTINISATION DU LAIT}

$\begin{array}{ccc}\text { JEAN VIEILLY } & \text { et } & \text { JEAN HARDER } \\ \begin{array}{c}\text { Doteur-Vétérinaire, Industriel laitier, } \\ \text { Grenoble. }\end{array} & \text { Chef d'exploitation de la Laiterie Moderne, } \\ & \text { (Fin.) } & \text { Grenoble. }\end{array}$

Donnons maintenant un aperçu des quelques observations faites au eours de nos essais sur les laits actinisés.

\section{ACTION DE L'ACTINISATION SUR LES LAITS}

L'actinisation n'altère pas l'aspect extérieur du lait. Aucune transformation n'est visible, aucune différenciation à ce sujet avec le lait cru, aussi bien de suite après l'actinisation qu'après un temps plus ou moins long.

L'examen organoleptique ne décèle aucun goût d'huile, et, de l'avis de tous ceux qui ont goûté ce lait, en comparaison avec du lait cru, en ignorant lequel était le lait actinisé, ce dernier fut toujours trouvé plus savoureux, plus sucré. Est-ce la raison pour laquelle les enfants le boivent plus facilement, et aussi le supportent mieux ? Nous en avons eu la preuve en faisant des essais sur un groupe d'enfants. Parmi ceux-ci se trouvait un hérédosyphilitique, type dont l'état était stationnaire et végétatif depuis plusieurs mois, et qui ne pouvait garder aucun lait. Le lait actinisé fut très bien accepté dès la première fois, et, après quinze jours, une amélioration sensible était visible, reprise de poids, air plus éveillé, joues colorées, etc.

Mais revenons à l'influence de l'actinisation sur le lait. La montée de la crème est la même que dans le lait cru. Quant à l'acidité, l'actinisation ne paraît pas devoir l'influencer. A ce sujet nous exprimerons toujours l'acidité en grammes par litre. Complètement d'aceord sur ce point avec le Professeur A. Monvoisin, il nous paraît plus logique, la densité et la matière grasse s'exprimant en grammes, d'employer la même unité pour l'acidité.

Si l'actinisation n'a pas d'influence quantitative sur l'acidité, elle en a une qualitative par contre, et sur laquelle nous reviendrons.

J. Proks (Le Lait, mars 1933, page 336), au cours des essais faits 